VERIFICATION OF AN ALTERNATIHG BIT PROTOCO. BY MEANS OF PROCESS A.GEBRA

J.A. BERGSTRA, J,W, KI.OP

Centre for Mathematics and Ccmputer Science

P.O.BOx 4079, $1009 \mathrm{AB}$ Amsterdam, The Netheriands

We verify a simple version of the alternating bit protocol in the system ACP (Algebra of Consunicating Processes with silent actions) augmented with Koomen's fair abstraction rule.

\title{
INTRODUCTION
}

Let $D$ be a finite set of data. These data are to be transmitted through an unreliable medium from location 1 to location 2 , by means of a transmission protocol T.

With $r 1$ (d) we denote the act of reading datum d at location 1 , whereas w2 (d) denotes the act of writing value $d$ at location 2 . The external (higher level) specification of the behaviour of $T$ is this:

$$
T=\sum_{d \in D} r 1(d) \cdot w 2(d) \cdot T
$$

Fron its initial state $T$ is enabled to read any $d \in D$, thereafter $T$ will write d at 2 and subsequently return to its initial state.

A very interesting mechanism to implement $T$ is the alternating bit protocol (from [21). This protocol turns out to be sufficiently complicated to serve as a test case for protocol verification methods (see HAILPERN \& OWICKI [7] and LAMPORT [8] for instance).

We will present a description and verification of ABP the altemating bit protocol), in terms of process algebra. Our presentation makes extensive use of $\mathrm{ACP}_{\tau}$, Algebra of Communicating Frocesses with silent actions, as well as of ideas by C.J. Koomen from philips Research.

The advantage of process algebra in contrast to techniques based on temporal logic and Hoare-style verification is mainiy that the entire verification is done in terms of calculations on the protocol itself. Both safety and liveness are simultaneously dealt with in the equational calculus of process algebra.

The structure of this note is as follows:

2. Explanation of the architecture of ABP.

2. Axioms and rules of process algebra.

3. Verification of $A B$.

This work was sponsored in part by ESPRI contract 432 METEOR. 
Remark. It must be said that $A B P$ as explained here is only one of the many variations on the same theme, and among these a rathex simple one. Process algebra is well suited to specify individual protocols; at present the specification of classes of protocols is not supported by process algebra. For other issues of a philosophical nature we refer to [10] and [11].

\section{ARCHITECTURE OF ABP}

1.1. The protocol can be visualised as follows:

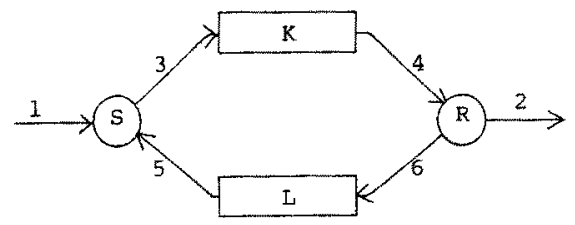

There are four components:

$S$ : sender. S reads data d at $1(d \in D)$, and communicates the data to channel

$\mathrm{K}$ until an acknowledgement has been received via channel $\mathrm{L}$.

$K:$ data transmission channel. $K$ communicates data in DOUDI (Di $=\{d i \mid d \in D\})$, and may communicate these correctly or communicate an error value $e . K$ is supposed to be fair in the sense that it will not produce an infinite consecutive sequence of errox outputs.

$R$ : receiver. $R$ receives data from $k$, outputs them at 2 and sends back acknowledgements via $\mathrm{L}$.

$I$ : acknowledgement transmission channel. The task of $L$ is to communicate boolean values from $R$ to $S$. The channel I may yield exror outputs but is also supposed to be fair.

The components $S, K, R$ and $L$ are processes. The protocol $T$ is described by

$$
\partial_{H}(S\|K\| R \| L)
$$

Here $\|$ denotes parallel composition and $\partial_{\mathrm{H}}$ encapsulates $\mathrm{S}\|\mathrm{k}\| \mathrm{F} \| \mathrm{L}$ by requiring that no extemal processes may interfere in the communications at ports $3,4,5$ and 6 .

In order to obtain an abstract view of the protocol the operator $\tau_{I}$ is applied, which replaces internal actions (in I) by the silent action $\tau$. Thus:

$$
T=\tau_{I} \partial_{H}(S\|K\| R \| L)
$$


Verification amounts to a proof that this $T$ satisfies the equation

$$
T=\sum_{d \in D} r I(d) . w 2(d) . T
$$

\subsection{Structure of the components of ABP.}

\subsubsection{Data and zctions.}

$D$ is the finite set of data that is to be transmitted by ABP. For $d \in D$, do and d are new data, obtained by appending 0 resp. 1 to d. We write:

$$
\begin{aligned}
& D O=\{d O \mid d \in D\} \\
& D I=\{d I \mid d \in D\} \\
& D=D \cup D O \cup D I \cup\{0,1, e\}
\end{aligned}
$$

$D$ is the set of data that occur as parameter of atomic actions.

For $t \in\{1, \ldots, 6\}$ there are read and write actions:

$x t(a), r e a d a \in D$ at $t$

wt $(a)$, write $a \in \mathbb{D}$ at $t$.

Here $t \in\{1, \ldots, 6\}$ is called a port (or location, but we prefer port).

Communication takes place at ports only:

$$
r t(a) \mid w t(a)=j \text {. }
$$

where $j$ is an internal action. Another kind of internal action is i. It corresponds to internal choices made by $K$ and $L$. The entire alphabet $A$ of proper actions is then as follows:

$$
A=\{x t(a) \mid i \leqslant t \leqslant 6, a \in \mathbb{D}\} \cup\{w t(a) \mid 1 \leqslant t \leqslant 6, a \in \mathbb{D}\} \cup\{i, j, \delta\} .
$$

The communication function . $1 .: A \times A \rightarrow A$ yields $\delta$ (deadlock or failure) except in the case mentioned before: $x t(a) \mid w t(a)=j$. of course the abstraction operator will introduce Milner's silent action $z$ and the universe of discourse consists of the processes over $A_{\tau}=A \cup\{\tau\}$. Furthermore $H$, the set of subatomic (or comunication) actions is:

$$
\bigcup_{t \in\{3,4,5,6\}} \bigcup_{a \in \mathbb{D}}\{r t(a), w t(a)\} .
$$

and $I$, the set of internal actions is just $\{1, j\}$.

\subsubsection{The individual components.}

We will first give the well-known state transition diagrams for "process graphs') for $S, K, L$ and $R$. Hexe a node is a state and an arrow denotes an action (i.e. state txansition of the process). Both state ana actions can be parametrised by data. 


\section{Channels:}

K:

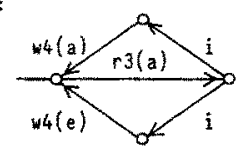

$$
K=\sum_{a \in D 0 u D 1} r 3(a) \cdot(i \cdot w 3(a)+i \cdot w 3(e)) \cdot K
$$

$(a \in D O \cup D L)$

L:

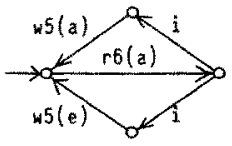$$
L=\sum_{a \in\{0,1\}} x 6(a) \cdot(1 . w 5(a)+i . w 5(e)) . L
$$

Note that $K$ and $L$, after receiving input, have a nondeterministic choice, by doing one of both $i$ actions.

At the level of this equational specification of $K$ and $E$ fairness is not yet mentioned. Fairness will come in when abstraction is applied to remove the i's.

\section{Sender:}

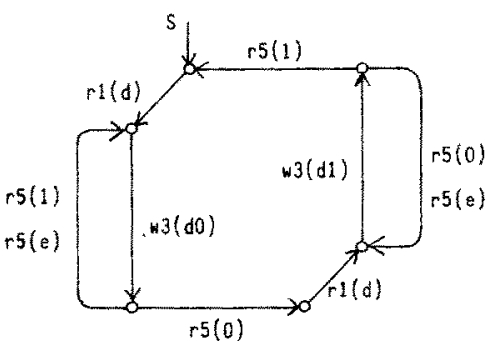

$$
\begin{aligned}
& s=s^{0} \cdot s^{1} \cdot s \\
& s^{n}=\sum_{d \in D} r 1(d) \cdot s_{d}^{n} \quad(n=0,1) \\
& s_{d}^{n}=w 3(d n) \cdot v_{d}^{n} \\
& v_{d}^{n}=(r 5(1-n)+r 5(e)) \cdot s_{d}^{n}+r 5(n)
\end{aligned}
$$




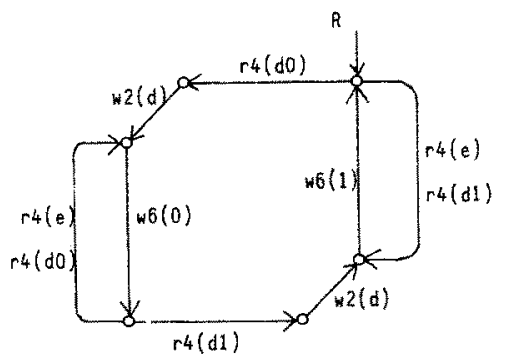

$$
\begin{aligned}
& R= R^{1} \cdot R^{0} \cdot R \\
& R^{n}=\left(\sum_{d \in D} r 4(d n)+r 4(e)\right) \cdot w 6(n) \cdot R^{n}+ \\
&+\sum_{d \in D} r 4(d(1-n)) \cdot w 2(d) \cdot w 6(1-n) \\
&(n=0,1)
\end{aligned}
$$

\section{PROCESS ALGEBRA}

\section{1. $\underline{\mathrm{ACP}}_{\tau}$.}

Let $A$ be a set of atomic actions and . 1. A A A $\rightarrow A$ a commuication function, which is commutative and associative and for which $\delta$ acts as a zero.

$A_{T}$ denotes $A \cup\{r\}$; $T$ is the silent action, that results from application of the abstraction operator.

The signature of operations of processes that we will use is this:

Table:.

\begin{tabular}{|c|l|}
\hline+ & alternative composition (sum) \\
\hline$\cdot$ & sequential composition (product) \\
\hline$U$ & parallel composition (merge) \\
\hline$U$ & left-merge \\
\hline$I$ & communication merge \\
\hline$\partial_{\mathrm{H}}$ & encapsulation \\
\hline$\tau_{\mathrm{I}}$ & abstraction \\
\hline$\delta$ & deadiock/failure \\
\hline$\tau$ & silent action \\
\hline
\end{tabular}


An $\mathrm{ACP}_{\tau}$ algebra is an algebra of the above signature (where | extends the communication function on atoms) and which satisfies the axioms in Table 2 . Here $H \subseteq A, I \subseteq A, \delta \notin I$ and $a, b, c$ range over $A$.

\begin{tabular}{|c|c|c|c|}
\hline \multicolumn{4}{|c|}{$A C P_{Y}$} \\
\hline$x+y=y+x$ & $A !$ & $x \tau=x$ & $\pi$ \\
\hline$x+(y+z)=(x+y)+z$ & $A 2$ & $\tau x+x=\tau x$ & 12 \\
\hline$x+x=x$ & A3 & $a(\tau x+y)=a(\tau x+y)+a x$ & 13 \\
\hline$(x+y) z=x z+y z$ & $A_{4}$ & & \\
\hline$(x y) z=x(y z)$ & A5 & & \\
\hline$x+\delta=x$ & $A 6$ & & \\
\hline$\delta x=\delta$ & A7 & & \\
\hline & & & \\
\hline$a|b=b| a$ & $\mathrm{Cl}$ & & \\
\hline$(a \mid b)|c=a|(b \mid c)$ & $\mathrm{C2}$ & & \\
\hline$\delta \mid \bar{a}=\delta$ & $\mathrm{C3}$ & & \\
\hline$x\|y=x\| y+y \mathbb{L} x+x \| y$ & cul & & \\
\hline $\mathrm{a}\lfloor x=a x$ & $\mathrm{CH} 2$ & $\tau \mathbb{L} x=\tau x$ & $T M I$ \\
\hline$(a x) \| y=a(x \| y)$ & $\mathrm{CH} 3$ & $(\tau x) \| y=\tau(x \| y)$ & $\operatorname{TM2}$ \\
\hline$(x+y) \mathbb{L} z=x \mathbb{L} z+y \mathbb{L} z$ & $\mathrm{CM}_{4}$ & $\tau \mid x=\delta$ & TC1 \\
\hline$(a x) / b=(a \mid b) x$ & CM5 & $x \mid \tau=\delta$ & $\mathrm{TC2}$ \\
\hline$a \mid(b x)=(a \mid b) x$ & CM6 & $(\tau x)|y=x| y$ & 103 \\
\hline$(a x) \|(b y)=(a \mid b)(x \| y)$ & CM7 & $x|(r y)=x| y$ & $T C 4$ \\
\hline$\{x+y)|z=x| z+y \mid z$ & CHB & & \\
\hline \multirow[t]{3}{*}{$x|(y+z)=x| y+x \mid z$} & CMg & & \\
\hline & & $a_{H}(\tau)=\tau$ & DI \\
\hline & & $\tau_{I}(\tau)=\tau$ & TII \\
\hline$a_{H}(a)=a$ if $a \notin H$ & 01 & $\tau_{1}(a)=a$ if $a \notin 1$ & 112 \\
\hline$a_{H}(a)=\delta$ if $a \in H$ & 02 & $\tau_{I}(a)=\tau$ if $a \in I$ & tI3 \\
\hline$a_{H}(x+y)=\partial_{H}(x)+\partial_{H}(y)$ & 03 & $\tau_{I}(x+y)=\tau_{I}(x)+\tau_{I}(y)$ & TI4 \\
\hline$a_{H}(x y)=a_{H}(x) \cdot a_{H}(y)$ & 04 & $\tau_{I}(x y)=\tau_{I}(x) \cdot \tau_{I}(y)$ & 115 \\
\hline
\end{tabular}

Table 2. 
$\mathrm{ACP}_{\tau}$ algebras satisfy the combinatorial identities shared by finite processes. In order to deal with infinite processes we will further assume that the following second order principles and rules are satisfied in the process algebra in which we model ABP, the alternating bit protocol.

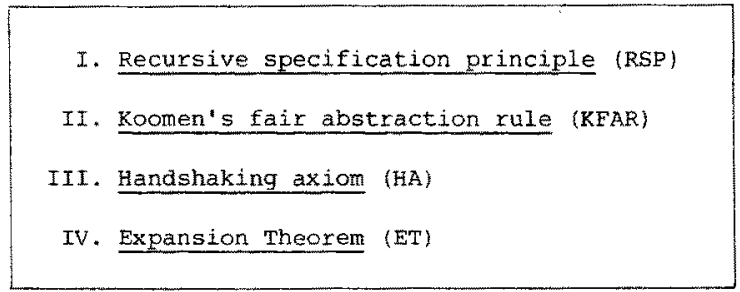

We will explain I-IV below. First, however, we allow ourselves some methodological remarks.

Remark 1. At present it is not possible to provide a renotely complete axiomatisation of processes that is of use "in general". But the equational (sub) systems $A C R$ and $A C P_{\tau}$ are a fixed kemel. Here ACP consists of the axioms Al-7,C1-3,CMl-9,D1-4, i.e. the left column of Table 2.

Remark 2. The system $A C P$ was introduced in [3], and $A C P_{C}$ was introduced in [4]. We view $\mathrm{ACP}_{T}$ as a reformulation of the basic issues of Milner's CCS [9] comments on the relation between $\mathrm{ACP}_{\tau}$ and CCS are in [4].

Remark 3. Koomen's fair abstraction rule has been derived from an idea that C.J. Koomen and R. Schutten used in experimental work on protocol verification. At Philips Research Eindhoven they have developed a formula manipulam tion package based on CCs.

\subsection{Explanation of the principles I,II,III, IV.}

\subsubsection{The recursive specification principle.}

Let $X, Y, X_{i}, Y_{i}$ (iE $\omega$ ) be variables for processes. We write $X$ for $\left\{X_{i}\{i \in \omega\}\right.$ and $Y$ for $\left\{Y_{i} \mid i \in \omega\right\}$. If $z$ is a collection of variables then $t(Z)$ denotes an $\mathrm{ACP}_{\mathrm{z}}$ term over $\mathrm{z}$.

Let $E \subseteq A$. We call the term $t(2)$ E-guarded if each variable in $t(Z)$ is preceded by an atom in $E$ and $t(Z)$ does not contain an operator $\tau_{I}$. Here 'preceded' is defined thus: if $t$ is a subterm (occurrence) in $x$ and $s$ in $y$, then $t$ precedes $s$ in $x \cdot y$; likewise in $x \| y$ (but not in $x \| y$ ). Example: $a \cdot(X \| Y)+b \cdot X$ and $a \cdot X \cdot(X \| Y)+z(b\lfloor X)$ are $(a, b)$-guarded, but $\tau . X+b . Y, \tau . X \mid a y$ and $(a X|| Y) \| b Z$ are not E-guarded for any $E$. We call an equation $X=t(Z)$ E-guarded if $t(Z)$ is E-guarded. 
Remark. The reason for excluding operators $\tau_{I}$ in an E-guarded term is that it is problematic to find a suitable definition of guardedness in the presence of such operators. Here 'suitable' refers to our wish to obtain unique processes (by RSP; see below) as solutions of systems of guarded equations. E. . . the following system has infinitely many solutions:

$$
\begin{aligned}
& x=a \cdot \tau_{(b)}(X) \\
& Y=b \cdot c_{\{a\}}(X) .
\end{aligned}
$$

Namely, every $x=a \cdot p$ with $a, b$ not in the alphabet of $p$ is a solution.

DEFINITION. A recursive specification $S_{E}(X ; X)$ is a collection of E-guarded equations (over ACP):

$$
x_{i}=t_{i}(X)
$$

together with an equation

$$
x=t(X)
$$

Remark. If $P_{*} P_{i}(i \in \omega)$ satisfy the system of equations $S_{E}\left(P_{i} P_{i} \mid\right.$ if $\left.\omega\right)$ then we want to view $s_{E}\left(X_{;} x_{i} \mid j \in \omega\right)$ as a specification of $P$ involving auxiliary processes $P_{i}$ (it $\left.\omega\right)$.

of course this definition incluaes the case of a finite specification.

The recursive specification principle (RSP) states that a recursive definition singles out a unique process (if any). In more formal notation:

$$
\text { (RSP) } \frac{s_{E}(X ; X) \quad s_{E}(Y, Y)}{X=Y}
$$

\subsubsection{Koomen's Eair abstraction rule (KFAR).}

This rule allows to compute $\tau_{I}(X)$ for certain $x$, thereby expressing the fact that certain steps in I will be fairly scheduled in such a way that eventually a step outside $I$ is performed. This is the fommal description of KFAR:

$$
(\mathrm{KFAR}) \frac{\forall \mathrm{n} \in \mathbb{Z}_{k} x_{n}=i_{n} \cdot x_{n+1}+Y_{n} \quad\left(i_{n} \in I\right)}{\tau_{I}\left(X_{n}\right)=\tau \cdot \tau_{I}\left(Y_{0}+\ldots+Y_{k-1}\right)}
$$


Here $\mathbb{Z}_{k}=\{0, \ldots, k-1\}$ and addition in subscripts works modulo $k$.

We illustrate the effect of KFAR in two simple examples:

(i) Suppose $x=i . x+$ where $a$ I. Then an application of KFAR yields:

$\tau_{I}(x)=\tau_{\text {a }}$. This expresses the fact that, due to some fairness mechanism, $i$ resists being performed infinitely many times consecutively.

(ii) Let $Y=i . Y$, then $\tau_{I}(Y)=\tau . \delta$. To see this note that $Y=i . Y+\delta$ and apply KFAR.

For a different approach to fairness in processes we refer to DE BAKKER \& ZUCKER [1].

\subsubsection{Axioms of standard concurrency.}

We will adopt the following axioms of "standard concurrency"; all axioms (1)-(6) hold for finite processes from $\mathrm{ACP}_{\tau}$. In [5] these axions are proved simultaneously with induction on term formation; we will only need here axioms $(3)-(6)$.
(1) $(x \| y)\|z=x\|(y \| z)$
(2) $(x \mid a y) \cup z=x \mid(a y \amalg z)$
(3) $x|y=y| x$
(4) $x\|y=y\| x$
(5) $x|(y \mid z)=(x \mid y)| z$
(6) $x\|(y \| z)=(x \| y)\| z$

\subsubsection{Handshaking axiom (HA).}

The handshaking axiom expresses the fact that all communications axe binary. i.e. work by means of handshaking.

$$
\text { (HA) } \quad x|Y| z=\delta
$$

\subsubsection{Expansion Theorem (ET).}

This theorem, in the context of CCS due to MINNER [9] and for ACP formulated in $[4]$, can be shown for finite processes from $A C P_{T}$. (see 151.) The Expansion Theorem presupposes $H A$ and the axiome of standard concurrency (except $(1),(2))$. The following notation is used: Let $x_{1}, \ldots, x_{k}$ be processes. With $X^{i}$ we denote merge of $a 11 \mathrm{x}_{n}$ such that $n \in\{1, \ldots, k\}-\{i\}$. With $X^{i, j}$ we denote the merge of ail $x_{n}$ such that $n \in\{1, \ldots, k\}-\{i, j\}$.

ET is then formulated as follows (for $k \geqslant 3$ ): 
(ET) $\quad x_{1}\|\ldots\| x_{k}=\sum_{1 \leqslant i \leqslant k} x_{i}\left\|X^{i}+\sum_{1 \leqslant i<j \leqslant k}\left(x_{i} \mid x_{j}\right)\right\| x^{i, j}$

ET is an indispensable tool for the calculation of terms of the form $x_{2}\|\ldots\| x_{k}$. Essentially it is a generalisation of the axiom CMl of $A C P_{\tau}$.

3. A VERTFICATION OF ABP

Let $T^{* *}=\sum_{d \in D} x 1(d) . w 2(d) . T^{*}$ and $T=\tau_{I} \partial_{H}(S\|K\| L \| R)$ in the notation of section 1. Section 1 fixes a set of atomic actions $A$ and a communication function on it.

Using $\mathrm{ACP}_{T}+\mathrm{RSP}+\mathrm{KFAR}+\mathrm{HA}+\mathrm{ET}$ we will show: $\mathrm{T}=\mathrm{T}^{*}$. Stated different1y:

$$
\tau_{I H} \partial_{H}(S\|K\| L \| R)=\sum_{d \in D} r 1(d) \cdot W^{2}(d) \cdot \tau_{I H} \partial_{H}(S\|K\| L \| R)
$$

For the proof we use the following notation:

$$
\left(x_{1}\left\|x_{2}\right\| x_{3} \| x_{4}\right)=\left(\begin{array}{c|c}
x_{1} & x_{2} \\
\hline x_{3} & x_{4}
\end{array}\right)
$$

Using this notation we have:

$$
T=\tau_{I} \partial_{H}\left(\frac{S}{L} \mid \frac{K}{R}\right)=\tau_{I} \partial_{H}\left(\begin{array}{c|c}
S^{0} \cdot S^{1} \cdot S & K \\
\hline L & R^{1} \cdot R^{0} \cdot R
\end{array}\right) .
$$

For $\mathrm{b} \in\{0,1\}$ we write

$$
T^{b}(X, Y)=\tau_{I} a_{H}\left(\begin{array}{c|c}
S^{b} \cdot S^{1-b} \cdot X & K \\
\hline L & R^{1-b} \cdot R^{b} \cdot y
\end{array}\right)
$$

In particular, $T=T^{\circ}(S, R)$.

CLAIM:

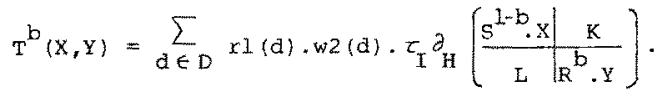

The claim proves $T=T^{*}$ as follows:

$$
T=T^{0}(S, R)=\sum_{d \in D} r I(d) \cdot w 2(d) \cdot \tau_{I} d_{H}\left(\begin{array}{c|c}
s^{l} \cdot S & K \\
\hline L & R^{0} \cdot R
\end{array}\right)=
$$




$$
\begin{aligned}
& \sum_{d \in D} I I(d) \cdot w 2(d) \cdot \tau_{I} \partial_{H}\left(\begin{array}{l|l}
S^{1} \cdot S^{0} \cdot S^{1} \cdot S & K \\
\hline L & R^{0} \cdot R^{1} \cdot R^{0} \cdot R
\end{array}\right)= \\
& \sum_{d \in D} x I(d) \cdot w 2(d) \cdot T^{2}\left(s^{2} \cdot S, R^{0} \cdot R\right)=
\end{aligned}
$$

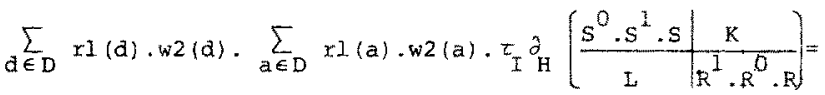

$$
\begin{aligned}
& \sum_{d \in D} r I(d) \cdot w 2(d) \cdot \sum_{a \in D} x I(a) \cdot w 2(a) \cdot T .
\end{aligned}
$$

Thus $T$ satisfies an (A-guarded) recursion equation which is also satisfied by $\mathrm{T}^{*}$. It follows by RSP that $\mathrm{T}=\mathrm{T}^{*}$.

\section{PROOF OF THE CLAIM. We write}

$$
G^{b}(X, Y)=\partial_{H}\left(\begin{array}{c|c}
s^{b} \cdot s^{1-b} \cdot X & x \\
\hline I & R^{1-b} \cdot R^{b} \cdot Y
\end{array}\right)
$$

and

$$
G_{d}^{b}(X, y)=\partial_{H}\left[\begin{array}{c|c}
s_{d}^{b} \cdot s^{1-b} \cdot x & k \\
\hline L & R^{1-b} \cdot R^{b} \cdot Y
\end{array}\right]
$$

Terms like $G^{b}(X, Y)$ and $G_{d}^{b}(X, Y)$ can be rewritten using the Expansion rheorem. ET will yield $4+6=10$ terms and in all cases in this proof at most 2 of these terms are not equal to $\delta$. In the sequel we will use applications of ET as a single calculation step. (Note that it is entirely feasible to verify all these applications of ET automatically.)

Now:

$$
\begin{aligned}
T^{b}(X, Y)= & \sum_{d \in D} r l(d) \cdot \tau_{I} \partial_{H}\left(\begin{array}{c|c|c}
s^{b} \cdot s^{1-b} \cdot x & K \\
\hline L & R^{1-b} \cdot K^{b} \cdot Y
\end{array}\right)= \\
& \sum_{d \in D} r 1(d) \cdot \tau_{I} G_{d}^{b}(X, Y) .
\end{aligned}
$$

We will derive a recursive specification for $G_{d}^{b}(X, Y)$ :

$$
\begin{aligned}
& G_{d}^{b}(X, Y)=j \cdot \partial_{H}\left(\begin{array}{c|c}
U_{a^{b}}^{b} \cdot s^{1-b} \cdot X & (i \cdot w 4(e)+i \cdot w 4(d b)) \cdot K \\
\hline L & R^{1-b} \cdot R^{b} \cdot Y
\end{array}\right)=
\end{aligned}
$$

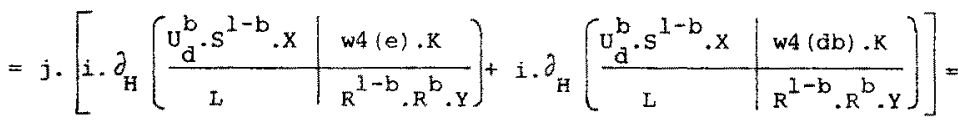




$$
\begin{aligned}
& =j \cdot\left[i \cdot j \cdot \partial_{H}\left(\begin{array}{c|c}
U_{d}^{b} \cdot s^{1-b} \cdot x & k \\
\hline I & w 6(1-b) \cdot R^{1-b} \cdot R^{b} \cdot Y
\end{array}\right]+\right. \\
& \left.+i \cdot j \cdot \partial_{H}\left(\begin{array}{c|c}
U_{d}^{b} \cdot s^{1-b} \cdot x & k \\
\hline L & \text { W2 (d) } \cdot w 6(b) \cdot R^{b} \cdot Y
\end{array}\right)\right]= \\
& =j \cdot\left[i \cdot j \cdot j \cdot \partial_{H}\left(\begin{array}{l|c}
u_{d}^{b} \cdot s^{1-b} \cdot x & k \\
\hline(i \cdot w 5(e)+i \cdot w 5(1-b)) \cdot L & R^{1-b} \cdot R^{b} \cdot y
\end{array}\right]+i \cdot j \cdot z\right] \\
& \text { (with } z=\partial_{H}\left(\begin{array}{c|c}
U_{d}^{b} \cdot s^{1-b} \cdot x & k \\
\hline L & \text { w2 (d) . w6 (b) . R } \cdot x
\end{array}\right),= \\
& =j \cdot\left[i \cdot j \cdot j \cdot \left\{i \cdot \partial_{H}\left(\begin{array}{c|c}
0^{b} \cdot s^{1-b} \cdot x & K \\
\hline w 5(e) \cdot L & R^{1-b} \cdot R^{b} \cdot Y
\end{array}\right)+\right.\right. \\
& \left.+i \cdot \partial_{H}\left(\begin{array}{c|c}
U_{a^{b}}^{b} \cdot s^{1-b} \cdot x & K \\
\hline W 5(1-b) \cdot L & R^{1-b} \cdot R^{b} \cdot Y
\end{array}\right)+i \cdot j \cdot z\right]= \\
& =j \cdot\left[i \cdot j \cdot j \cdot \left\{i \cdot j \cdot \partial_{H}\left(\begin{array}{c|c}
s_{d}^{b} \cdot s^{L-b} \cdot x & K \\
\hline L & R^{I-b} \cdot R^{b} \cdot x
\end{array}\right]+\right.\right. \\
& \left.\left.+i \cdot j \cdot \partial_{H}\left[\begin{array}{c|c}
s_{d^{b} \cdot s^{1-b} \cdot x} & k \\
\hline L & R^{1-b} \cdot R^{b} \cdot x
\end{array}\right]\right\}+i \cdot j \cdot z\right]= \\
& =j \cdot\left[i \cdot j \cdot j \cdot i \cdot j \cdot G_{d}^{b}(X, Y)+i \cdot j \cdot z\right] \text {. }
\end{aligned}
$$

We can now apply KFAR for $k=6$ and $y_{0}=\delta, x_{1}=i \cdot j \cdot z, y_{2}=\ldots=x_{5}=\delta$.

This gives:

$$
\tau_{I}\left(G_{d}^{b}(X, Y)\right)=\tau \cdot \tau_{I}(i \cdot j \cdot z) .
$$


Hence:

$$
\begin{aligned}
& T^{b}(X, Y)=\sum_{d \in D} r 1(d) \cdot \tau_{I}\left(G_{d}^{b}(X, Y)\right)=\sum_{d \in D} r I(d) \cdot \tau \cdot \tau_{I}(i \cdot j \cdot Z)= \\
& =\sum_{d \in D} r l(d) \cdot \tau \cdot \tau \cdot \tau \cdot \tau_{I}(z)=\sum_{d \in D} r l(d) \cdot \tau_{I}(z)= \\
& =\sum_{d \in D} r 1(a) \cdot \tau_{I} \partial_{H}\left[\begin{array}{c|c}
0^{b} \cdot s^{1-b} \cdot x & k \\
\hline L & w 2(d), w 6(b) \cdot R^{b} \cdot Y
\end{array}\right]= \\
& =\sum_{d \in D} r 1(d) \cdot \tau_{I}\left[w 2(a) \cdot \partial_{H}\left[\begin{array}{c|c}
u_{d^{b} \cdot s^{1-b} \cdot x} & k \\
\hline L & w 6(b) \cdot R^{b} \cdot x
\end{array}\right]=\right. \\
& =\sum_{d \in D} r I(d) \cdot w 2(d) \cdot \tau_{I}\left(K_{d}^{b}(X, Y) t\right. \\
& \left(\text { with } x_{d}^{b}(X, Y)=\partial_{H}\left(\begin{array}{c|c}
U_{d}^{b} \cdot s^{1-b} \cdot x & k \\
\hline I & w 6(b), R^{b} \cdot Y
\end{array}\right)\right) \text {. }
\end{aligned}
$$

The next part of the proof of the claim consists in deriving a recursion equation for $\mathrm{K}_{\mathrm{d}}^{\mathrm{b}}(\mathrm{X}, \mathrm{Y})$ :

$$
\begin{aligned}
& k_{d}^{b}(X, Y)=j \cdot \partial_{H}\left(\begin{array}{l|l}
U_{a^{b}}^{b} s^{2-b} \cdot x & k \\
\hline(i, w 5(b)+1 \cdot w 5(e)) \cdot L & R^{b}, Y
\end{array}\right)= \\
& =j \cdot\left[\text { i. } \partial_{H}\left(\begin{array}{c|c}
u_{a^{b}}^{b} \cdot s^{1-b} \cdot x & k \\
\hline w 5(b) \cdot L & R^{b} \cdot Y
\end{array}\right]+i \cdot \partial_{H}\left[\begin{array}{c|c}
u_{a^{b}}^{b} s^{1-b} \cdot x & K \\
\hline w 5(e) \cdot L & R^{b} \cdot X
\end{array}\right]=\right. \\
& =j \cdot\left[i \cdot j \cdot \partial_{H}\left(\begin{array}{c|c}
s^{1-b} \cdot x & k \\
\hline L & R^{b} \cdot Y
\end{array}\right]+i \cdot j \cdot \partial_{H}\left(\begin{array}{c|c}
s_{d}^{b} \cdot s^{1-b} \cdot x & K \\
\hline L & R^{b} \cdot Y
\end{array}\right]=\right. \\
& =j \cdot\left[i \cdot j \cdot v+i \cdot j \cdot j \cdot \partial_{H}\left(\begin{array}{c|c}
0_{d^{b}}^{b} \cdot s^{1-b} \cdot x & (i \cdot w 4(e)+i \cdot w 4(a b)) \cdot K \\
\hline I & R^{b} \cdot Y
\end{array}\right]\right.
\end{aligned}
$$




$$
\begin{aligned}
& \text { (with } v=\partial_{H}\left(\begin{array}{c|c}
s^{1-b} \cdot x & k \\
\hline L & R^{b} . Y
\end{array}\right),= \\
& =j \cdot\left[i \cdot j \cdot v+i \cdot j \cdot j \cdot\left\{i \cdot \partial_{H}\left(\begin{array}{c|c}
u_{a}^{b} \cdot s^{1-b} \cdot x & w 4(e) \cdot k \\
\hline L & R^{b} \cdot x
\end{array}\right]+\right.\right. \\
& \left.+i \cdot \partial_{H}\left\{\begin{array}{c|c}
U_{d}^{b} \cdot s^{L-b} \cdot X & w 4(d b) \cdot K \\
\hline L & R^{b} \cdot Y
\end{array}\right]\right\}= \\
& =j \cdot\left[i \cdot j \cdot v+i \cdot j \cdot j \cdot\left\{i \cdot j \cdot \partial_{H}\left[\begin{array}{c|c}
u_{d}^{b} \cdot s^{I-b} \cdot x & K \\
\hline L & w 6(b) \cdot R^{b} \cdot x
\end{array}\right]+\right.\right. \\
& \left.\left.+\dot{i} \cdot j \cdot \partial_{H}\left(\begin{array}{c|c}
u_{d}^{b} \cdot s^{l-b} \cdot x & k \\
\hline L & w \in(b) \cdot R^{b} \cdot Y
\end{array}\right)\right\}\right]= \\
& =j \cdot\left[i \cdot j \cdot v+i \cdot j \cdot j \cdot j \cdot j \cdot k_{d}^{b}(x, y)\right] \cdot
\end{aligned}
$$

Applying KFAR we get:

$$
\begin{aligned}
& \tau_{I}\left(k_{d}^{b}(x, Y)\right)=\tau \cdot \tau_{I}(i, j \cdot V)=\tau \cdot \tau \cdot \tau \cdot \tau_{I}(V)=\tau \cdot \tau_{I}(V)= \\
& \tau \cdot \tau_{I} \partial_{H}\left(\begin{array}{ll|l}
s^{1-b} \cdot x & K \\
\hline L & R^{b} \cdot Y
\end{array}\right) .
\end{aligned}
$$

We conclude:

$$
\begin{aligned}
& T^{b}(x, Y)=\sum_{d \in D} r 1(d) \cdot w 2(d) \cdot \tau_{I}\left(K_{d}^{b}(x, Y)\right)= \\
& =\sum_{d \in D} r I(d) \cdot w 2(d) \cdot \tau \cdot \tau_{I} \partial_{H}\left(\begin{array}{c|c}
S^{1-b} \cdot x & K \\
\hline L & R^{b} \cdot Y
\end{array}\right)=
\end{aligned}
$$




$$
=\sum_{d \in D} r l(d) \cdot w 2(d) \cdot \tau_{I} \partial_{H}\left(\begin{array}{c|c}
s^{l-b} \cdot x & K \\
\hline L & R^{b} \cdot Y
\end{array}\right) .
$$

This finishes the proof of the clain and the verification of $A B P$.

\section{REFBRENCE9}

[1] DE BAKKER, J.W. \& J.I. ZUCKER, Compactness in semontios for merge and faip merge, Report IW 238/83, Mathematisch Centrum Amsterdam 1983.

[2] BARTLETT, K.A., R.A. SCANTLEBURY \& P.T. WILKINSON, A note on reliable full-duples tranomission over hatf duplex lines, CACM 12, No.5 (1969).

[3] BERGSTRA, J.A. \& J.W. KLOP, Frocess Algebra for Synchronous Communication, Information and Control, Vol.60, Nos.1-3, 1984, 109-137.

[4] BERGSTRA, J.A. J.W. KIOP, Algebra of Comminicating Processes, to appear in Proc. of the CWI symposium Mathematics and Computer science (eđs. J.W. de Bakker, M. Hazewinkel and J.K. Lenstra), North-Holland, Ansterdan 1985.

[5] BERGSTRA, J.A. \& J.W. KLOP, Algebra of Comminicating Processes with abstraction, to appear in Theoretical Computer science, 1985.

[6] HAILPERN, B.T., Vemifying concurrent processes using temporal logic. Springer LNCS 129, 1982.

[7] HALEERN, B.T. \& S. OWICKI, Vemfying network protocole using temporal logie, in: Trends and applications symposium, National Bureat of standards 1980 .

[8] LAMPORT, L., Snecifying concurrent program modules, ACM Toplas, vol.5, No.2, P.190-222.

[9] MTLNER, R., A Calculus of Commuicating Systems, springex LNCS 92, 1980.

[10] SCHWARTZ, R.I. \& P. MELIAR SMITH, From state machine to temperat Zogic, specification methods for protocol standards, IEEE Transactions or communication, Vo1.30, No.12 (1982) p.2486-2496.

[II] YEMINI, Y. \& J.F. KUROSE, Can current protocol perifieation techriques guarantee comectness? Computer networks, Vol.6, No.6 (1982), p. $377-381$. 